\title{
AN ACTION OF A FINITE GROUP ON AN $n$-CELL WITHOUT STATIONARY POINTS
}

\author{
BY E. E. FLOYD AND R. W. RICHARDSON ${ }^{1}$ \\ Communicated by Deane Montgomery, November 19, 1958
}

If $G$ is a transformation group on a space $X$, then $x \in X$ is a stationary point if $g x=x$ for every $g \in G$. It has been an open problem, proposed by Smith [5] and by Montgomery [1, Problem 39], to determine whether every compact Lie group acting on a cell or on Euclidean space has a stationary point. Smith $[4 ; 5]$ has shown the answer to be in the affirmative in case $G$ is a toral group or a finite group of prime power order. In this note we give a simplicial action of $A_{5}$, the group of even permutations on five letters, on an $n$-cell without stationary points. Greever [3] has recently shown that the only finite groups of order less than 60 which could possibly act simplicially on a cell without stationary points are a certain class of groups of order 36.

We wish to thank P. E. Conner for his help and encouragement.

1. The coset space $S O(3) / I$. Let $S O(3)$ denote the group of all proper rotations of Euclidean 3 -space $E^{3}$ and let $I \subset S O(3)$ be the group of rotational symmetries of the icosahedron. As a group, $I$ is isomorphic to $A_{5}$ (see [9, pp. 16-18]) and hence is simple.

LEMma 1. The coset space $S O(3) / I$ has the integral homology groups of the 3-sphere $S^{3}$.

Proof. Let $Q$ denote the algebra of quaternions and $Q_{1} \subset Q$ the group of quaternions of norm one. Identify $Q$ with $E^{4}$ and $Q_{1}$ with $S^{3}$. Let $\tau: Q_{1} \rightarrow S O(3)$ be the standard homomorphism, which is a two-toone covering map. Set $I^{\prime}=\tau^{-1}(I)$. Then $\tau$ induces a homeomorphism $Q_{1} / I^{\prime} \approx S O(3) / I$.

The natural map $\pi: Q_{1} \rightarrow Q_{1} / I^{\prime}$ is a covering map and the group of covering translations is given by the action of $I^{\prime}$ on $Q$, by right multiplication. Since every covering translation preserves orientation it follows that $Q_{1} / I^{\prime}$ is an orientable 3-manifold and hence $H_{3}\left(Q_{1} / I^{\prime}\right)$ $\approx H_{3}(S O(3) / I) \approx Z$ (here $Z$ denotes the integers).

From covering space theory the fundamental group $\pi_{1}\left(Q_{1} / I^{\prime}\right)$ is isomorphic to $I^{\prime}$. Thus $H_{1}\left(Q_{1} / I^{\prime}\right)$ is isomorphic to $I^{\prime} /\left[I^{\prime}, I^{\prime}\right]$ where $\left[I^{\prime}, I^{\prime}\right]$ denotes the commutator subgroup of $I^{\prime}$. Since $I$ is simple,

1 The first named author is the holder of a NSF Senior Postdoctoral Fellowship; the work of the second has been supported in part by contract A.F. 49(638)-104. 
$[I, I]=I$. Also $\tau$ maps $\left[I^{\prime}, I^{\prime}\right]$ onto $[I, I]$; it follows that either $\left[I^{\prime}, I^{\prime}\right]=I^{\prime}$ or $\left[I^{\prime}, I^{\prime}\right] \approx I$. But $Q_{1}$ contains only one element of order two. Since $I$ contains fifteen elements of order two, $\left[I^{\prime}, I^{\prime}\right]$ is not isomorphic to $I$. Thus $I^{\prime}=\left[I^{\prime}, I^{\prime}\right]$ and $H_{1}\left(Q_{1} / I^{\prime}\right)=0$. By Poincare duality it follows that $H_{2}\left(Q_{1} / I^{\prime}\right)=0$. The lemma follows.

2. Action of $I$ on $S O(3) / I$. Let $I$ act on $S O(3) / I$ by $g_{1} \cdot(g I)=g_{1} g I$. A point $\dot{g}=g I$ of $S O(3) / I$ is fixed under this action if and only if $g$ belongs to the normalizer of $I$ in $S O(3)$. But $I$ is a maximal finite subgroup of $S O(3)$ (see [9, pp. 16-18]); furthermore, $I$ is not included in any nonfinite proper closed subgroup of $S O(3)$, since this is not the case for the only two classes of such subgroups. Since $I$ is not normal, it follows that $I$ is its own normalizer. Hence there is exactly one stationary point of this action, and this is $\dot{e}$.

We say that the transformation group $G$ acts simplicially on the space $X$ if there exists a triangulation of $X$ with respect to which the homeomorphism $g: X \rightarrow X$ is simplicial for every $g \in G$.

Lemma 2. The action of $I$ on $S O(3) / I$ is simplicial.

Proof. Let $I^{\prime} \times I^{\prime}$ act on $Q\left(=E^{4}\right)$ by the rule $\left(q_{1}, q_{2}\right) \cdot q=q_{1} q q_{2}^{-1}$. This represents $I^{\prime} \times I^{\prime}$ as a finite group of orthogonal transformations of $E^{4}$. Hence we may find a triangulation of $S^{3}\left(=Q_{1}\right)$ such that the action of $I^{\prime} \times I^{\prime}$ is simplicial. The method is similar to one used by Whitney [8, p. 358, Lemma 3b]; we omit the details.

Now $e \times I^{\prime}$ acts simplicially on $Q_{1}$, and the orbit space is $Q_{1} / I^{\prime}$. By taking a barycentric subdivision, the triangulation of $Q_{1}$ induces a triangulation of the orbit space $Q_{1} / I^{\prime}$. The action of $I^{\prime} \times e$ on $Q_{1}$ induces an action of $I^{\prime} \times e$ on $Q_{1} / I^{\prime}$ and since $I^{\prime} \times e$ acts simplicially on $Q_{1}$ the induced action is simplicial with respect to the induced triangulation of $Q_{1} / I^{\prime}$.

In the action of $I^{\prime} \times e\left(=I^{\prime}\right)$ on $Q_{1} / I^{\prime}$ the effective group is $I^{\prime} /$ kernel $\tau$. Furthermore the homeomorphism $\tau_{1}$ of $Q_{1} / I^{\prime}$ on $S O(3) / I$ is equivariant with respect to the action of $I^{\prime} /$ kernel $\tau$ on $Q_{1} / I^{\prime}$ and the action of $I$ on $S O(3) / I$. It follows that the action of $I$ on $S O(3)$ is simplicial.

3. Action of $I$ on a cell. We may assume that the triangulation of $Q_{1}$ is $C^{1}$ in the sense of [6] and that $e$ is a vertex. Since

$$
\tau_{1} \cdot \pi: Q_{1} \rightarrow S O(3) / I
$$

is a $C^{1}$-map the induced triangulation of $S O(3) / I$ is a $C^{1}$ triangulation. It follows that the closed star of the point $I$ of $S O(3) / I$ is a 3-cell (see [6, p. 818, Theorem 5]). Let $K$ denote the complex resulting if 
we remove the open star of the point $I$ from $S O(3) / I$, and let $|K|$ denote the corresponding space. Then $|K|$ is acyclic (i.e. $H_{i}(|K|)=0$ for $i>0$, and $\left.H_{0}(|K|) \approx Z\right)$, and $I$ acts simplicially on $|K|$ without stationary points.

Consider now the join $L=K \circ I$ of the complex $K$ and the complex $I$, where $I$ is the complex consisting of 60 vertices (the points of $I$ ) and no simplices of higher dimension. Since $I$ acts on $K$, and $I$ acts on $I$ (by left multiplication), then $I$ acts simplicially on $L$. In fact, $g \in I$ maps a line segment from $x \in K$ to $h \in I$ linearly into the line segment from $g(x)$ to $g h$. Furthermore, there are no stationary points on $L$. The polyhedron $|L|$ is a union of 60 cones over $|K|$, each pair intersecting in $|K|$. It follows that $|L|$ is acyclic, and also simply connected.

Let $\left(v_{1}, \cdots, v_{n}\right)$ denote the set of vertices of $L$. Each $g \in I$ induces a permutation $\eta_{g}$ of the vertices of $L ; \eta_{g}$ may be considered as an element of the full symmetric group $S_{n}$ on $n$ letters.

Let $e_{1}, \cdots, e_{n}$ be basis vectors for $E^{n}$. Each element $n$ of $S_{n}$ determines a permutation of $\left(e_{1}, \cdots, e_{n}\right)$. If we extend linearly, $n$ defines a linear transformation of $E^{n}$. This defines an action of $S_{n}$ as a group of linear transformations of $E^{n}$.

Triangulate $E^{n}$ so that the action of $S_{n}$ is simplicial, and so that the simplex spanned by $e_{1}, \cdots, e_{n}$ is a simplex of the triangulation. Define an embedding $f$ of $L$ in $E^{n}$ by setting $f\left(v_{i}\right)=e_{i}$ and extending $f$ linearly to each simplex. Then $f$ is equivariant. Hence $I$ acts on $f(L)$, and without stationary points.

Let $F_{I}$ be the set of points of $E^{n}$ which are stationary under the action of $I$. Then $F_{I} \cap f(L)=\varnothing$. If we take sufficiently fine barycentric subdivisions we may assume that $F_{I}$ does not intersect the first closed regular neighborhood of $f(L)$ (see [2, pp. 70-72 for definitions]), denoted by $N(f(L))$. Since $I$ acts simplicially on $E^{n}$ and $f(L)$ is invariant, it follows that $N(f(L))$ is also invariant. Since $f(L)$ is simply connected and acyclic, it follows from a theorem of J. H. C. Whitehead [7, Corollary 3, p. 298] that the regular neighborhood is a combinatorial $n$-cell. Thus $I$ acts simplicially on the combinatorial $n$-cell $N(f(L))$ without stationary points.

\section{BiBLIOGRAPHY}

1. S. Eilenberg, On the problems of topology, Ann. of Math. vol. 50 (1949) pp. 247260.

2. S. Eilenberg and N. Steenrod, Foundations of algebraic topology, Princeton, 1952.

3. J. J. Greever, Fixed points of finite transformation groups, Dissertation, University of Virginia. 
4. P. A. Smith, Fixed point theorems for periodic transformations, Amer. J. Math. vol. 63 (1941) pp. 1-8.

5. - Stationary points of transformation groups, Proc. Nat. Acad. Sci. U.S.A. vol. 28 (1942) pp. 293-297.

6. J. H. C. Whitehead, On C complexes, Ann. of Math. vol. 41 (1940) pp. 809824.

7. - Simplicial spaces, nuclei, and m-groups, Proc. London Math. Soc. vol 45 (1939) pp. 243-327.

8. H. Whitney, Geometric integration theory, Princeton, 1957.

9. H. Zassenhaus, Theory of groups, New York, 1949.

UNIVERSITY OF VIRGINIA AND

InSTITUTE For AdVANCEd STUdy

UNIVERSITY OF Michigan AND

Princeton University 